DR. ANDREY ABRAMOV (Orcid ID : 0000-0002-7646-7235)

Article type : Original Article

\title{
Variability of mitochondrial energy balance across brain regions
}

XinPing Chen ${ }^{1,2 *}$, Andrey Y. Vinokurov ${ }^{3 *}$, Evgeniy A. Zherebtsov,3,4, Olga A. Stelmashchuk ${ }^{3}$, Plamena R. Angelova ${ }^{1}$, Noemi Esteras ${ }^{1}$, Andrey Y. Abramov ${ }^{1}$, 3\#

${ }^{1}$ Department of Clinical and Movement Neurosciences, UCL Queen Square Institute of Neurology, Queen Square, London, WC1N 3BG, UK

${ }^{2}$ CAS Key Laboratory of Brain Function and Disease, School of Life Sciences, University of Science and Technology of China, Hefei 230027, Anhui, China

${ }^{3}$ Cell Physiology and Pathology Laboratory, Orel State University, Orel, 302026, Russia

${ }^{4}$ Optoelectronics and Measurement Techniques Laboratory, University of Oulu, Oulu, 90014, Finland

*Joint fist authors

\# Corresponding author: Prof Andrey Y. Abramov a.abramov@ucl.ac.uk

This article has been accepted for publication and undergone full peer review but has not been through the copyediting, typesetting, pagination and proofreading process, which may lead to differences between this version and the Version of Record. Please cite this article as doi: 10.1111/JNC.15239

This article is protected by copyright. All rights reserved 


\section{Abstract}

Brain is not homogenous and neurons from various brain regions are known to have different vulnerability to mitochondrial mutations and mitochondrial toxins. However, it is not clear if this vulnerability is connected to different energy metabolism in specific brain regions. Here, using live cell imaging we compared mitochondrial membrane potential and NADH redox balance in acute rat brain slices in different brain regions and further detailed the mitochondrial metabolism in primary neurons and astrocytes from rat cortex, midbrain and cerebellum. We have found that mitochondrial membrane potential $(\Delta \Psi \mathrm{m})$ is higher in brain slices from hippocampus and brain stem. In primary co-cultures, $\Delta \Psi_{\mathrm{m}}$ in astrocytes was lower than in neurons, while in midbrain cells it was higher than in cortex and cerebellum. The rate of NADH production and mitochondrial NADH pool were highest in acute slices from midbrain and midbrain primary neurons and astrocytes. Although the level of ATP was similar among primary neurons and astrocytes from cortex, midbrain and cerebellum, the rate of ATP consumption was highest in midbrain cells that lead to faster neuronal and astrocytic collapse in response to inhibitors of ATP production. Thus, midbrain neurons and astrocytes have a higher metabolic rate and ATP consumption that makes them more vulnerable to energy deprivation.

List of abbreviations: ATP - adenosine tri phosphate; HBSS - Hanks' Balanced Salt solution; FCCP - Carbonyl cyanide-p-trifluoromethoxyphenylhydrazone; IAA -iodoacetic acid; NADH - Nicotinamide adenine dinucleotide; NADPH -Nicotinamide adenine dinucleotide phosphate; Rh123 - rhodamine 123; TCA - tricarboxylic acid cycle; TMRM tetramethylrhodamine; $\Delta \Psi \mathrm{m}-$ mitochondrial membrane potential 


\section{Introduction}

Despite the fact that the brain is relatively small compared to the rest of the body (1$1.5 \%$ ), is one of the most energy-consuming organs and utilises more oxygen and glucose than any other tissue. The major energy demands of the brain are due to neuronal signaling ( $70 \%$ is used for calcium signalling, action and resting potentials, glutamate cycling) and the remaining $30 \%$ of the energy expenses are associated with non-signaling activities (de novo synthesis of proteins, phospholipids, oligonucleotide turnover, axonal transport, actin cytoskeleton remodelling, etc.) (Dienel 2019). Importantly, the rate of information processing in the brain is also limited by efficient energy production (Harris et al. 2012; Attwell \& Laughlin 2001). Due to the tight equilibrium between energy production and consumption, the brain is extremely vulnerable to any alterations of this balance, as occurs for example in an ischemic condition.

In the brain, energy in the form of ATP is mainly produced by oxidative phosphorylation in the mitochondria, however, to a lesser degree ATP is also produced by glycolysis. Due to anatomical specificity (astrocytes have a closer access to the blood vessels), the energy balance in neurons is mostly relying on astrocytic support. Considering this, neurons and astrocytes have a distinctive energy metabolism. Additionally, due to differences in electrophysiological activity and de novo synthesis of proteins, phospholipids etc., the rate of energy consumption in these cells should also be different.

Neurological conditions that lead to neuronal cell death, such as ischemia (stroke) or epilepsy, despite the complexity of their ethiopathological mechanism, are high energy demanding processes (Tambasco et al. 2018; Abramov \& Duchen 2010; Kovac et al. 2012). All neurodegenerative disorders have shown to have energy metabolism or/and mitochondrial dysfunction involved in the mechanism of development of pathology (Abramov \& Angelova 2019). It should be also noted that mitochondrial toxins (rotenone and MPP+) induce neuronal loss in specific brain regions initiating Parkinson's disease

(Singer \& Ramsay 1990; Hartley et al. 1994), or changes in frataxin leading to Friederich's Ataxia (Abeti et al. 2016; Lodi et al. 2002). However, the reason for the specific vulnerability of neurons in particular brain regions to mitochondrial toxins or to mitochondrial dysfunction is not clear. One of the possible explanations could be the 
distinctive rate of energy production or consumption in these areas. A number of approaches have been used for the detection of different parameters of energy metabolism including ATP levels and the rate of glucose metabolism (Kleinridders et al. 2018). Despite that the mitochondrial membrane potential $\left(\Delta \Psi_{m}\right)$ should have some defined? range values to act as a proton-motive force for ATP production, the level of $\Delta \Psi \mathrm{m}$ can generally vary in cells and this can have effect on some signalling processes, e.g. $\mathrm{Ca}^{2+}$ uptake and reactive oxygen species production (Yao et al. 2011; Esteras et al. 2017). However, it is not clear how $\Delta \Psi_{\mathrm{m}}$ and mitochondrial metabolism vary between neurons and astrocytes in different brain regions, and how fast these cells consume ATP.

Here, we studied differences in $\Delta \Psi \mathrm{m}$ and $\mathrm{NADH}$ redox index in acute live brain slices from different brain regions. Using primary co-cultures of neurons and astrocytes from cortex, midbrain and cerebellum we studied the differences in the $\Delta \Psi \mathrm{m}, \mathrm{NADH}$ redox balance, ATP level and the rate of ATP consumption between neurons and astrocytes from these brain regions.

\section{Materials and methods}

The study was not pre-registered. No blinding was performed.

\subsection{Acute brain slices}

All animal work for isolation and preparation of brain slices was approved by the Institutional ethical committee of Orel State University ((Minutes No. 18 dated 21.02.2020) in compliance with Russian Federation legislation. The rats were grouphoused and maintained on a 12-h light cycle (lights on 07:00) and had ad libitum access to water and food. The experiments were conducted on male Wistar rats (12 for all preparations) at 12 weeks of age $(250-300 \mathrm{~g})$. The animals were euthanized by cervical dislocation. After isolation, the rat brain was placed in chilled HBSS. Horizontal sections of different brain regions (hippocampus, cortex, cerebellum, brain stem) with a thickness of $500 \mu \mathrm{m}$ were cut -, according to a standard procedures (Angelova \& Muller 2006; Novikova et al. 2020) and stored prior to experiments for at least 1 hour $/ 37^{\circ} \mathrm{C}$

\subsection{NADH and Rh123 fluorescence measurements in acute brain slices}


To measure the mitochondrial membrane potential with Rh123 or the endogenous content of NADH in acute brain slices, the setup was assembled in two versions: with a BDL-SMN-375 laser source (Becker \& Hickl, Germany) for excitation of NADH fluorescence at a wavelength of $375 \mathrm{~nm}$ or LED M455F1 (Thorlabs Inc., USA) for excitation of Rh123 fluorescence at a wavelength of $490 \mathrm{~nm}$. In this system, the excitation radiation through the optical fiber passes through the collimator and the bandpass filter (when using LED M455F1), and then, through the beam splitting filter plate and the lens is directed to the studied area. Emitted fluorescence light was reflected through a $416 \mathrm{~nm}$ (in the configuration for NADH measurements) or $490 \mathrm{~nm}$ long-pass filters (in the configuration for Rh123) to a highly sensitive DCC 3260C cooling CCD camera (Thorlabs et al., USA). The field of view of the camera on the sample is a rectangular section with an area of about $1 \mathrm{~mm}^{2}$. The fluorescence imaging channel reflected from the light source through filters and a highly sensitive DCC 3260C cooling CCD camera is recorded (Thorlabs et al., USA). Brain slices were loaded with $1 \mu \mathrm{M}$ Rhodamine123 (Rh123) (Molecular Probes; catalog \# R22420) for 20 min before experiments. Rh123 has a slower permeability for plasmalemmal membrane compared to other cationic indicators that makes it a better candidate to be used in de-quench mode which is less dependent on the changes of the focal plane.

\subsection{Primary co-cultures of neurons and astrocytes}

Mixed cultures of cortical, midbrain and cerebellar granule neurones and glial cells were prepared as described previously (Maiolino et al. 2019) with modifications, from SpragueDawley rat pups (22 either sex for all cell preparations) 2-4 days post-partum (UCL breeding colony). Pups were euthanized by cervical dislocation. Experimental procedures were performed in compliance with the United Kingdom Animals (Scientific Procedures) Act of 1986 and with the European directive 2010/63/EU. Cerebellum, cortex and midbrain were isolated from 2 pups and moved into ice-cold $\mathrm{HBSS}\left(\mathrm{Ca}^{2+}, \mathrm{Mg}^{2+}\right.$ free, Invitrogen, Paisley, UK; Catalog \#14175046). The tissue was minced and trypsinised $\left(0.1 \%\right.$ for $15 \mathrm{~min}$ at $\left.37^{\circ} \mathrm{C}\right)$, triturated and plated on poly-D-lysine-coated coverslips and cultured in Neurobasal A medium (Gibco-Invitrogen, Paisley, UK; catalog \# 21103049) supplemented with B-27 (Gibco-Invitrogen, Paisley, UK; Catalog \# 17504044) and Lglutamine. Cultures were maintained at $37^{\circ} \mathrm{C}$ in a humidified atmosphere of $5 \% \mathrm{CO}_{2}$ and 
95\% air, fed twice a week and maintained for a minimum of 12 days before experimental use to ensure the expression of glutamate and other receptors. Neurons were easily distinguishable from glia: they appeared phase bright, had smooth rounded somata and distinct processes, and lay just above the focal plane of the glial layer. Cells were used after days 12-15.

\subsection{Live cell imaging}

Measurement of cellular free magnesium using the $\mathrm{Mg}^{2+}$-sensitive fluorescent probe Mag-Fura-2 can be used as an indirect indicator of ATP consumption/production (Ludtmann et al. 2016). Mag-Fura-2 AM (Invitrogen Catalog number \# M1291) dye was added with $0.005 \%$ pluronic acid for 30 min in HBSS solution (Invitrogen catalog \# 14025092). The energy capacity of the cell was assessed as the time between application of inhibitors and the time of cell lysis. Fluorescence measurements were obtained on an epifluorescence inverted microscope equipped with a 20x fluorite objective. $\left[\mathrm{Mg}^{2+}\right]_{c}$ was monitored in single cells using excitation light provided by a Xenon arc lamp, the beam passing monochromator at 340 and $380 \mathrm{~nm}$ (Cairn Research, Kent, UK). Emitted fluorescence light was reflected through a $515 \mathrm{~nm}$ long-pass filter to a cooled CCD camera (Retiga, Qlmaging, Canada) and digitised to 12-bit resolution. All imaging data were collected and analysed using software from Andor (Belfast, UK).

NADH autofluorescence was measured using an epifluorescence inverted microscope equipped with $a \times 20$ fluorite objective. Excitation light at a wavelength of $360 \mathrm{~nm}$ was provided by a Xenon arc lamp, the beam passing through a monochromator (Cairn Research, Faversham, Kent, UK). Emitted fluorescence light was reflected through a 455 $\mathrm{nm}$ long-pass filter to a cooled CCD camera (Retiga, QImaging, Surrey, BC, Canada) and digitised to 12 bit resolution (Al-Menhali et al. 2020). Imaging data were collected and analysed using Andor iQ3 imaging software (Belfast, UK).

Confocal images were obtained using Zeiss 710 CLSM microscope equipped with a META detection system and a $40 \times$ oil immersion objective. Mitochondrial membrane potential was identified using the potential sensitive indicator tetramethylrhodamine (TMRM) (Invitrogen catalog number \# T668). Cells were loaded for $40 \mathrm{~min}$ at room temperature and superfused with $25 \mathrm{nM}$ TMRM, excited at $565 \mathrm{~nm}$ and imaged with a 
$580 \mathrm{~nm}$ emission filter as previously described (Angelova et al. 2018; Bartolome et al. 2017). Measurements of TMRM fluorescence in neurons and astrocytes were determined across z-tacks. Illumination intensity was kept to a minimum (at $0.1-0.2 \%$ of the max laser output) to avoid phototoxicity and the pinhole set to give an optical slice of $\sim 2 \mu \mathrm{m}$.

To determine the ATP levels, primary co-cultures were transfected with a mitochondriallytargeted ATP probe (AT1.03) generated by Imamura et al. (Imamura et al. 2009), using Effectene (Qiagen) according to the manufacturer's instructions. The FRET was quantified by the $527: 475 \mathrm{~nm}$ ratio after an excitation with $405 \mathrm{~nm}$ and a filtered at 515 to $580 \mathrm{~nm}$ (Ludtmann et al. 2014).

\subsection{Statistical analysis}

Results are expressed as means \pm SEM (standard error of the mean); one-way ANOVA with post-hoc Tukey's HSD correction for multiple comparisons and Student's t-test were used, where appropriate. Statistical analysis was performed using Origin 2019 (Microcal Software Inc., Northampton, MA, USA) software. Differences were considered to be significantly different if $p<0.05$. No exclusion criteria were pre-determined. No sample size calculation was performed, the number of rats was taken as a number of cell preparations or brain slices preparation. No test for outliers was conducted. Data were not assessed for normality

\section{Results}

Measurements of mitochondrial membrane potential in acute rat brain slices from different regions with Rhodamine123 showed that $\Delta \Psi_{\mathrm{m}}$ is not equal between them (Figure 1A). To avoid any misinterpretations of the results, $1 \mu \mathrm{M}$ FCCP was added at the end of each experiment to induce depolarisation that led to redistribution of the Rh123 from mitochondria to the cytosol and was registered as an increase in Rh123 fluorescence. When the indicator diffused out through the plasmalemmal membrane $t$ could be seen from the slow decrease in Rh123 fluorescence after the FCCP-induced peak (Figure $1 \mathrm{~B}-\mathrm{C}$ ). The highest values of $\Delta \Psi_{\mathrm{m}}$ were observed in hippocampal slices $\left(155 \pm 7 \%\right.$ of cortical $\Delta \Psi_{\mathrm{m}} ; \mathrm{N}=7$ rats, $\mathrm{n}=14$ slices; Figure $\left.1 \mathrm{~A}, \mathrm{~B}\right)$ and in slices from brain stem $(195 \pm 11 \% ; N=7$ rats, $n=14$ slices; Figure $1 \mathrm{~A})$. Mitochondrial membrane potential in 
cells of midbrain slices was also higher compared to the cortex $(127 \pm 7 \%$; $N=7$ rats, $n=14$ slices; Figure $1 \mathrm{~A}$ ) while $\Delta \Psi_{\mathrm{m}}$ in cerebellar slices was similar to cortices (Figure $1 \mathrm{~A}, \mathrm{C}$ ).

Mitochondrial membrane potential might be different due to a number of factors including activity of the electron transport chain of the mitochondria, the level of uncoupling proteins, or the mechanism of maintenance of $\Delta \Psi \mathrm{m}$. To find out what causes the differences in the activity of mitochondrial electron transport chain in different brain regions, we measured NADH level in various parts of acute brain slices.

\section{NADH redox state in acute brain slices from different regions}

$\mathrm{NADH}$ is a major donor of electrons in mitochondrial complex I and measurements of NADH autofluorescence can give information about the activity of mitochondrial respiration in live cells. To distinguish mitochondrial NADH fluorescence from NADPH signal and non-mitochondrial NADH, we maximised respiration in brain slices by adding $1 \mu \mathrm{M}$ FCCP (which minimises mitochondrial NADH level $-0 \%$ ) followed by inhibition of respiration by $1 \mathrm{mM} \mathrm{NaCN}$, that blocks consumption of $\mathrm{NADH}$ and maximally increases NADH level in mitochondria (Figure $2 \mathrm{~A}-\mathrm{B}$ ). The difference in NADH between minimal and maximal values could be taken as a mitochondrial NADH pool. Interestingly, mitochondrial NADH pool in midbrain cells was significantly higher compared to mitochondrial NADH level in cells from cortical, hippocampal, brain stem and cerebellar slices (Figure 2B-G).

NADH redox level reflects the balance between NADH production and NADH consumption in mitochondria. The lowest level we observed in hippocampal $(41 \pm 3 \% ; \mathrm{N}=5$ rats, $\mathrm{n}=15$ slices) and cerebellar ( $38 \pm 3 \%, N=5$ rats, $n=15$ slices) slices suggests higher respiratory activity in these brain regions. The highest NADH redox level was observed in midbrain $(62 \pm 5 \%, N=5$ rats, $n=14$ slices) and brain stem $(58 \pm 5 \%, N=5$ rats, $n=14)$ slices (Figure $2 \mathrm{~B}-\mathrm{F}, \mathrm{H}$ ). Inhibition of the mitochondrial respiration with $\mathrm{NaCN}$ completely blocks the consumption of NADH in complex I and the rate of rise of NADH could be accepted as the rate of $\mathrm{NADH}$ production in tricarboxylic acid cycle (Krebs cycle) (Figure 2B for explanation - see also Figure $2 \mathrm{~B}-\mathrm{F}, \mathrm{I}$ ). The rate of NADH production was different in slices from various brain regions with highest values in midbrain and hippocampus (figure 
21). Considering that the highest rate of NADH production is found in the mitochondria of cells from midbrain slices (Figure 2l), the elevated NADH redox level in this region could be explained by the higher production rate, while in brain stem it more likely due to a lower respiratory activity.

Interestingly, the level of NADPH and non-mitochondrial NADH (which was measured as the autofluorescence insensitive to FCCP and $\mathrm{NaCN}$ ) was also highest in the midbrain area (Figure 2J) and the brain stem. Non-mitochondrial NADH/ NADPH levels in the brain stem and the cerebellum were higher compared to the cortical or the hippocampal areas (Figure 2J).

Broad distribution of the signal of $\Delta \Psi_{\mathrm{m}}$ and $\mathrm{NADH}$ parameters in brain slices can be explained by the short lifetime of ex vivo models and assessment of bioenergetics in different brain regions could not be complete without understanding the interaction between neurons and astrocytes in primary co-cultures. Considering this, we produced primary co-cultures of neurons and astrocytes from the most vulnerable regions to ischaemia and mitochondrial toxins - cortex, midbrain and cerebellum and compared the major bioenergetic characteristics between cells and brain regions.

\section{$\Delta \Psi \mathrm{m}$ is higher in neurons compared to astrocytes in the co-cultures}

In primary co-cultures neurons and astrocytes are distributed in different layers and very often are pooled in measurements of mitochondrial membrane potential. Using Z-stacks we were able to separate the signals and compared $\Delta \Psi_{\mathrm{m}}$ in neurons and astrocytes (Figure $3 \mathrm{~A}$ ). In all studied primary co-cultures - cortical, midbrain and cerebellar, mitochondrial membrane potential in neurons was significantly higher than in astrocytes. Thus, the maximal difference in $\Delta \Psi_{\mathrm{m}}$ between cell types was observed in cortical cocultures where the level of TMRM fluorescence in astrocytes was significantly smaller (Figure 3B; $51 \pm 4 \%$ of neuronal, $\mathrm{N}=5$ experiments, $\mathrm{n}=155$ cells; $\mathrm{p}<0.001$ ). In cerebellar and midbrain co-cultures $\Delta \Psi \mathrm{m}$ of astrocytes was also low $-58 \pm 5 \%$ and $67 \pm 7 \%$ of neighbouring neurons, respectively (Figure 3C-D; $N=7$ experiments).

Despite the difference in the $\Delta \Psi_{\mathrm{m}}$ between neurons and astrocytes, the mechanism of maintenance of mitochondrial membrane potential is similar for both type of cells. Thus, application of the inhibitor of $F_{0}-F_{1}-A T P s y n t h a s e$ oligomycin $(2 \mu \mathrm{g} / \mathrm{ml})$ to midbrain co- 
culture of neurons and astrocytes induced an increase in TMRM fluorescence by $\sim 20 \%$ in both type of cells, that strongly suggests that $\Delta \Psi_{\mathrm{m}}$ is not maintained by hydrolysis of ATP in the $F_{0}-F_{1}$-ATPase ( $N=4$ experiments; Figure $3 \mathrm{E}$ ). Subsequent application of the inhibitor of complex I rotenone $(1 \mu \mathrm{M})$ led to profound mitochondrial depolarisation with relatively small effect of the uncoupler FCCP $(1 \mu \mathrm{M})$ confirming that $\Delta \Psi_{\mathrm{m}}$ in neurons and astrocytes is maintained by the electron transport chain (Figure 3E).

\section{$\Delta \Psi_{m}$ in primary neurons and astrocytes in midbrain is higher than in cortical or cerebellar cells}

Midbrain neurons had the highest mitochondrial membrane potential (124 $\pm 8 \%$ of cortical neurons, Figure 3F) while primary neurons from cerebellum -the lowest one $(78 \pm 8 \%$ of cortical; Figure 3F). Similar difference between cells from the three brain regions was also observed for astrocytes (Figure $3 G$ ). Thus, difference in $\Delta \Psi_{\mathrm{m}}$ between midbrain and cortical astrocytes was even higher $(141 \pm 10 \%$ in midbrain, Figure $3 \mathrm{E})$ and lower in cerebellar astrocytes (62 $\pm 6 \%$ of cortical astrocytes, Figure $3 G)$.

\section{Mitochondrial NADH level in primary co-cultures}

Mitochondrial NADH level and NADH pool in primary co-cultures was assessed in the same way as in experiments with acute brain slices (Figure 2), but in these experiments we were able to distinguish autofluorescence of $\mathrm{NADH}$ from neurons and astrocytes (Figure 4 A-B, C).

Despite the dramatic difference in $\Delta \Psi_{\mathrm{m}}$ between neurons and astrocytes, NADH redox level, which mostly corresponds to the rate of respiration in these cells, was not significantly different between astrocytes and neurons in co-cultures from cortex, midbrain and cerebellum (Figure 4B-C, D-E). Importantly, despite the potential difference in the mechanisms of bioenergetics between these cells, mitochondrial pool was also similar between astrocytes and neurons in cortical, midbrain and cerebellar primary cocultures that in combination with redox state suggests equal production of NADH in the TCA cycle (Figure $4 \mathrm{~F}$ ). However, redox index in midbrain neurons $(52 \pm 4, n=130$ cells) and astrocytes $(48 \pm 4, n=149$ cells) was significantly lower compared to cells from the cerebellum (75 $\pm 3, n=210$ astrocytes; $71 \pm 2, n=158$ neurons) or the cortex $(55 \pm 2, n=170$ astrocytes; $58 \pm 2, n=139$ neurons) (Figure 4 C-D) that is in agreement with the results of 
$\Delta \Psi_{\mathrm{m}}$ measurements in these cells from these regions. Interestingly, NADH pool in primary neurons and astrocytes from the cortex was lower compared to cerebellum and midbrain (Figure $4 \mathrm{E}-\mathrm{F}$ ).

\section{Intracellular ATP level in primary co-cultures of neurons and astrocytes}

Intracellular level of ATP was assessed using the mitochondrially-targeted FRET probe AT1.03 (Imamura et al. 2009). The basal level of ATP was different between neurons and astrocytes and this difference was dependent on the brain regions (Figure 5). Thus, cortical astrocytes have a higher $[\mathrm{ATP}]_{\mathrm{m}}$ compared to neighbouring neurons $(110.3 \pm 4.3 \%$ of neuronal; Figure $5 \mathrm{~A}$ ). In midbrain astrocytes the level of ATP was almost similar to neuronal $(96+1.9 \%$; Figure $5 \mathrm{~A})$ while in cerebellar co-culture astrocytic $[A T P]_{\mathrm{m}}$ was lower than in neurons $(89 \pm 4.1 \%$; Figure $5 \mathrm{~A})$. Interestingly, the basal ATP level was similar between astrocytes from cortical, midbrain and cerebellum (Figure $5 \mathrm{D}$ ). Cerebellar neurons in our experiments had the highest ATP level (130\% of cortical, $N=4$; Figure E) and in midbrain neurons it was also relatively high $-118 \pm 4 \%$ of cortical.

\section{ATP consumption in primary co-cultures}

The basal level of ATP is an important characteristic of the cell energy metabolism, but it reflects the balance between ATP production and consumption in resting cells at a certain point. To identify the rate of ATP consumption of neurons and astrocytes from different brain regions, we estimated the levels of cellular free magnesium using the indicator Mag-Fura-2 in the presence of inhibitors of the ATP production.

$\mathrm{Mg}^{2+}$ is released from the Mg-ATP complex upon hydrolysis of ATP, and therefore, changes in the fluorescence of Mag-Fura-2 can be used as an indirect probe for ATP consumption (Leyssens et al. 1996). Mag-fura-2 is also a low affinity $\mathrm{Ca}^{2+}$ indicator, and this feature allowed us to detect the cell collapse caused by the inability to maintain the ionic homeostasis upon ATP depletion, which appears as a sudden increase in the MagFura-2 fluorescence due to a massive calcium influx.

Co-application of inhibitors of oxidative phosphorylation $(2 \mu \mathrm{g} / \mathrm{ml}$ oligomycin) and glycolysis $(20 \mu \mathrm{M}$ iodoacetic acid IAA) blocks the main sources of ATP production, and 
from that point cells can only consume the ATP already available in the cells. This allows us to estimate the rate of ATP consumption independently of the production.

Interestingly, although neurons are thought to be more energy-demanding cells compared to astrocytes, inhibition of ATP synthesis led to a faster energy depletion and cell lysis in astrocytes compared to neurons (Figure 5D-F). This effect was observed for cortical, midbrain and cerebellum co-cultures (Figure 5F).

The midbrain neurons from primary co-cultures were the most vulnerable to inhibition of ATP production and showed the shortest time ( $69 \pm 5 \mathrm{~min}, \mathrm{~N}=4$ experiments, $\mathrm{n}=87$ cells) to energy collapse, compared to cortical ( $96 \pm 8$ min, $N=5$ experiments, $n=103$ cells) or cerebellar neurons ( $94 \pm 7$ min; $N=4$ experiments, $n=97$ cells; Figure 5D-F).

\section{Discussion}

Although production of ATP in mitochondria requires a very narrow range of proton motive force and values mitochondrial membrane potential, correspondingly (Mitchell 1961), here we found that $\Delta \Psi_{\mathrm{m}}$ is highly variable across cell types (neurons and astrocytes) and brain regions. Considering the similar levels of ATP in these cells (Figure 5) we suggest that the difference in $\Delta \Psi_{\mathrm{m}}$ is dependent on the rate of metabolism and mechanism of maintenance of mitochondrial membrane potential. Additionally, $\Delta \Psi \mathrm{m}$ is important for mitochondrial calcium uptake (Abeti \& Abramov 2015) and the difference between neurons and astrocytes may reflect the high importance of calcium buffering in mitochondria for neurons.

Higher demands of midbrain neurons for ATP availability have been suggested before (Surmeier et al. 2017), and explained by the difference in ATP consumption rates between $\mathrm{Na}^{+} / \mathrm{K}^{+}$ATPase and $\mathrm{Ca}^{2+}$-ATPase. In agreement with this, we have found that midbrain neurons consume ATP much faster compared to cortical and cerebellar neurons. Although we have not separated the signal from more specialised neurons (such a dopaminergic, striatal, etc.) due to technical difficulties, the variety of responses of neurons to ATP production inhibition can suggest that some specialised neurons consume ATP faster than other types of neurons. Interestingly, despite the presence of 
electrical activity of neurons compared to astrocytes (Verkhratsky et al. 2017), astrocytes from different brain areas consumed ATP faster and thus collapsed faster compared to neurons, despite the similar basal level of ATP (Figure 5). This is an interesting observation and could be explained by the supporting function of astrocytes towards neurons and could further elucidate why the astrocyte-neuron interaction is vitally important in the development of pathology of neurological diseases (Angelova \& Abramov 2014).

Basal ATP level is similar for most of the cells except for cerebellar neurons and the rate of ATP consumption is higher in midbrain neurons and astrocytes. Thus, NADH pool in these cells, together with the rate of $\mathrm{NADH}$ production were elevated, and were associated with similar or higher rate of mitochondrial respiration (NADH redox index, Figure 2 and 4). Importantly, this increased rate of respiration and metabolism was not associated with mitochondrial uncoupling because the $\Delta \Psi_{\mathrm{m}}$ was also high in primary midbrain neurons and astrocytes. Thus observed higher rate of ATP consumption and higher consumption rate of mitochondrial complex I substrates might potentially explain why this brain region is particularly vulnerable to the action of mitochondrial toxins and pesticides and severe bioenergetic disbalance might contribute to trigger dopaminergic neurodegeneration in Parkinson's disease (Schapira et al. 1989; Betarbet et al. 2000; Abramov \& Angelova 2019).

--Human subjects --

Involves human subjects:

If yes: Informed consent \& ethics approval achieved:

=> if yes, please ensure that the info "Informed consent was achieved for all subjects, and the experiments were approved by the local ethics committee." is included in the Methods.

ARRIVE guidelines have been followed:

Yes

$\Rightarrow>$ if it is a Review or Editorial, skip complete sentence $=>$ if No, include a statement in the "Conflict of interest disclosure" section: "ARRIVE guidelines were not followed for the following reason:

"

(edit phrasing to form a complete sentence as necessary).

$\Rightarrow$ if Yes, insert in the "Conflict of interest disclosure" section: 
"All experiments were conducted in compliance with the ARRIVE guidelines." unless it is a Review or

Editorial

Conflicts of interest: none

$\Rightarrow$ if 'none', insert "The authors have no conflict of interest to declare."

$=>$ otherwise insert info unless it is already included

\section{Acknowledgements}

This work was supported by the grant of the Russian Federation Government $\mathrm{Nr}$. 075-15-2019-1877. E.Z. acknowledges the support of the Academy of Finland (Grant No. 318281).

\section{Declaration of competing interest}

The authors declare that they have no known competing financial interests or personal relationships that could have appeared to influence the work reported in this paper.

\section{References:}

Abeti, R. and Abramov, A. Y. (2015) Mitochondrial Ca(2+) in neurodegenerative disorders. Pharmacol Res 99, 377-381.

Abeti, R., Parkinson, M. H., Hargreaves, I. P., Angelova, P. R., Sandi, C., Pook, M. A., Giunti, P. and Abramov, A. Y. (2016) 'Mitochondrial energy imbalance and lipid peroxidation cause cell death in Friedreich's ataxia'. Cell Death Dis 7, e2237.

Abramov, A. Y. and Angelova, P. R. (2019) Mitochondrial dysfunction and energy deprivation in the mechanism of neurodegeneration. Turkish Journal of Biochemistry 44, 723-729.

Abramov, A. Y. and Duchen, M. R. (2010) Impaired mitochondrial bioenergetics determines glutamateinduced delayed calcium deregulation in neurons. Biochim Biophys Acta 1800, 297-304.

This article is protected by copyright. All rights reserved 
Al-Menhali, A. S., Banu, S., Angelova, P. R., Barcaru, A., Horvatovich, P., Abramov, A. Y. and Jaganjac, M. (2020) Lipid peroxidation is involved in calcium dependent upregulation of mitochondrial metabolism in skeletal muscle. Biochim Biophys Acta Gen Subj 1864, 129487.

Angelova, P. and Muller, W. (2006) Oxidative modulation of the transient potassium current IA by intracellular arachidonic acid in rat CA1 pyramidal neurons. Eur J Neurosci 23, 2375-2384.

Angelova, P. R. and Abramov, A. Y. (2014) Interaction of neurons and astrocytes underlies the mechanism of Abeta-induced neurotoxicity. Biochem Soc Trans 42, 1286-1290.

Angelova, P. R., Iversen, K. Z., Teschemacher, A. G., Kasparov, S., Gourine, A. V. and Abramov, A. Y. (2018) Signal transduction in astrocytes: Localization and release of inorganic polyphosphate. Glia 66, 2126-2136.

Attwell, D. and Laughlin, S. B. (2001) An energy budget for signaling in the grey matter of the brain. J Cereb Blood Flow Metab 21, 1133-1145.

Bartolome, F., Esteras, N., Martin-Requero, A. et al. (2017) Pathogenic p62/SQSTM1 mutations impair energy metabolism through limitation of mitochondrial substrates. Sci Rep 7, 1666.

Betarbet, R., Sherer, T. B., MacKenzie, G., Garcia-Osuna, M., Panov, A. V. and Greenamyre, J. T. (2000) Chronic systemic pesticide exposure reproduces features of Parkinson's disease. Nat Neurosci 3, 1301-1306.

Dienel, G. A. (2019) Brain Glucose Metabolism: Integration of Energetics with Function. Physiol Rev 99, 949-1045.

Esteras, N., Rohrer, J. D., Hardy, J., Wray, S. and Abramov, A. Y. (2017) Mitochondrial hyperpolarization in iPSC-derived neurons from patients of FTDP-17 with 10+16 MAPT mutation leads to oxidative stress and neurodegeneration. Redox Biol 12, 410-422.

Harris, J. J., Jolivet, R. and Attwell, D. (2012) Synaptic energy use and supply. Neuron 75, 762-777.

Hartley, A., Stone, J. M., Heron, C., Cooper, J. M. and Schapira, A. H. (1994) Complex I inhibitors induce dose-dependent apoptosis in PC12 cells: relevance to Parkinson's disease. J Neurochem 63, 19871990.

Imamura, H., Nhat, K. P., Togawa, H., Saito, K., lino, R., Kato-Yamada, Y., Nagai, T. and Noji, H. (2009) Visualization of ATP levels inside single living cells with fluorescence resonance energy transferbased genetically encoded indicators. Proc Natl Acad Sci U S A 106, 15651-15656.

Kleinridders, A., Ferris, H. A., Reyzer, M. L. et al. (2018) Regional differences in brain glucose metabolism determined by imaging mass spectrometry. Mol Metab 12, 113-121.

Kovac, S., Domijan, A. M., Walker, M. C. and Abramov, A. Y. (2012) Prolonged seizure activity impairs mitochondrial bioenergetics and induces cell death. J Cell Sci 125, 1796-1806.

This article is protected by copyright. All rights reserved 
Leyssens, A., Nowicky, A. V., Patterson, L., Crompton, M. and Duchen, M. R. (1996) The relationship between mitochondrial state, ATP hydrolysis, [Mg2+]i and [Ca2+]i studied in isolated rat cardiomyocytes. J Physiol 496 ( Pt 1), 111-128.

Lodi, R., Rajagopalan, B., Bradley, J. L. et al. (2002) Mitochondrial dysfunction in Friedreich's ataxia: from pathogenesis to treatment perspectives. Free Radic Res 36, 461-466.

Ludtmann, M. H., Angelova, P. R., Ninkina, N. N., Gandhi, S., Buchman, V. L. and Abramov, A. Y. (2016) Monomeric Alpha-Synuclein Exerts a Physiological Role on Brain ATP Synthase. J Neurosci 36, 10510-10521.

Ludtmann, M. H., Angelova, P. R., Zhang, Y., Abramov, A. Y. and Dinkova-Kostova, A. T. (2014) Nrf2 affects the efficiency of mitochondrial fatty acid oxidation. Biochem J 457, 415-424.

Maiolino, M., O'Neill, N., Lariccia, V., Amoroso, S., Sylantyev, S., Angelova, P. R. and Abramov, A. Y. (2019) Inorganic Polyphosphate Regulates AMPA and NMDA Receptors and Protects Against Glutamate Excitotoxicity via Activation of P2Y Receptors. J Neurosci 39, 6038-6048.

Mitchell, P. (1961) Coupling of phosphorylation to electron and hydrogen transfer by a chemi-osmotic type of mechanism. Nature 191, 144-148.

Novikova, I. N., Manole, A., Zherebtsov, E. A., Stavtsev, D. D., Vukolova, M. N., Dunaev, A. V., Angelova, P. R. and Abramov, A. Y. (2020) Adrenaline induces calcium signal in astrocytes and vasoconstriction via activation of monoamine oxidase. Free Radic Biol Med 159, 15-22.

Schapira, A. H., Cooper, J. M., Dexter, D., Jenner, P., Clark, J. B. and Marsden, C. D. (1989) Mitochondrial complex I deficiency in Parkinson's disease. Lancet 1, 1269.

Singer, T. P. and Ramsay, R. R. (1990) Mechanism of the neurotoxicity of MPTP. An update. FEBS Lett 274, $1-8$.

Surmeier, D. J., Obeso, J. A. and Halliday, G. M. (2017) Selective neuronal vulnerability in Parkinson disease. Nat Rev Neurosci 18, 101-113.

Tambasco, N., Romoli, M. and Calabresi, P. (2018) Selective basal ganglia vulnerability to energy deprivation: Experimental and clinical evidences. Prog Neurobiol 169, 55-75.

Verkhratsky, A., Zorec, R. and Parpura, V. (2017) Stratification of astrocytes in healthy and diseased brain. Brain Pathol 27, 629-644.

Yao, Z., Gandhi, S., Burchell, V. S., Plun-Favreau, H., Wood, N. W. and Abramov, A. Y. (2011) Cell metabolism affects selective vulnerability in PINK1-associated Parkinson's disease. J Cell Sci 124, 4194-4202.

This article is protected by copyright. All rights reserved 


\section{Figure Legends}

Figure 1. Mitochondrial membrane potential in acute slices from different brain regions

$\Delta \Psi_{\mathrm{m}}$ was measured using Rhodamine123 as a fluorescent indicator. A. $\Delta \Psi_{\mathrm{m}}$ in acute slices of different brain regions. For each brain region $N=7$ rats, $n=14$ slices. Rhodamine123 fluorescence in cortical slices was taken as $100 \%$. B-C Effect of $1 \mu \mathrm{M}$ FCCP on Rhodamine123 fluorescence in hippocampal and cerebellar slices. The error bars depict mean \pm SEM, statistical significance was calculated using one way ANOVA with post-hoc Tukey's HSD correction for multiple comparisons. ${ }^{*} p<0.05 ;{ }^{* *} p<0.01 ;{ }^{* * *} p$ $<0.001$.

Figure 2. Difference in mitochondrial NADH level and redox state in acute slices from brain regions. A. Representative images of NADH autofluorescence in cortical acute slices before and after application of $1 \mu \mathrm{M}$ FCCP and $1 \mathrm{mM} \mathrm{NaCN}$. Representative traces of NADH autofluorescence from acute slices from cortex (B), midbrain (C), hippocampus (D), cerebellum (E) and brain stem (F). Experiment in $\mathbf{A}$ is taken as example how different NADH parameters were calculated including mitochondrial NADH pool (G), NADH redox state $(\mathbf{H})$, rate of $\mathrm{NADH}$ increase after $1 \mathrm{mM} \mathrm{NaCN}$ application (I) and non-mitochondrial NADH and NADPH pool $(\mathbf{J})$ in acute slices. For each brain region measurements $-\mathrm{N}=5$ rats, $\mathrm{n}=14$ slices. The error bars depict mean $\pm \mathrm{SEM}$, statistical significance was calculated using ANOVA with the Tukey post-hoc test. ${ }^{*} p<0.05 ;{ }^{* *} p<$ $0.01 ;{ }^{* * *} p<0.001$.

Figure 3. Difference in $\Delta \Psi m$ between primary neurons and astrocytes isolated from cortex, midbrain and cerebellum.

A -Mitochondrial membrane potential in primary co-cultures was assessed using different Z-projections for separation of the TMRM signal from neurons and astrocytes and identification of the maximal fluorescence from mitochondria of live cells. Mitochondrial membrane potential is higher in neurons than in astrocytes in primary cortical $(B, N=5$ cell culture preparations), cerebellar $(\mathbf{C} ; \mathrm{N}=7$ cell culture preparations) and midbrain ( $\mathbf{D} ; \mathrm{N}=7$ 
cell culture preparations). TMRM was normalised as $100 \%$ to neurons. E-Measurement of TMRM fluorescence in response to applications of $2 \mu \mathrm{g} / \mathrm{ml}$ oligomycin, $1 \mu \mathrm{M}$ rotenone and $1 \mu \mathrm{M}$ FCCP to midbrain neurons and astrocytes. Difference in $\Delta \Psi_{\mathrm{m}}$ between neurons (F) and astrocytes (G) from primary co-cultures of cortex, midbrain and cerebellum. $\Delta \Psi_{\mathrm{m}}$ in astrocytes and neurons from cortical cultures were taken as $100 \%$. ${ }^{*} p<0.05 ;{ }^{* *} p<0.01 ;{ }^{* * *} p<0.001$.

Figure 4. Comparison of mitochondrial NADH redox level and NADH pools in primary neurons and astrocytes isolated from cortex, midbrain and cerebellum.

Representative images $(\mathrm{A})$ and traces of $\mathrm{NADH}$ autofluorescence in response to $1 \mu \mathrm{M}$ FCCP and $1 \mathrm{mM} \mathrm{NaCN}$ in cerebellar astrocyte (B) and midbrain neuron (C). Mitochondrial NADH redox indexes calculated for astrocytes (D) and neurons (E). F Difference in mitochondrial NADH pool between neurons and astrocytes from primary cocultures of cortex, midbrain and cerebellum. For experiments on primary neurons and astrocytes from each regions $\mathrm{N}=5$ cell culture preparations. ${ }^{*} \mathrm{p}<0.05 ;{ }^{* *} \mathrm{p}<0.01$; ${ }^{* * *} \mathrm{p}<$ 0.001 .

Figure 5. ATP levels and rate of ATP consumption in primary neurons and astrocytes isolated from cortex, midbrain and cerebellum

A-C Basal ATP levels were estimated in neurons and astrocytes after transfection of the genetically- encoded, mitochondrially-targeted probe AT1.03 as described in Methods. For experiments on primary neurons and astrocytes from each regions $\mathrm{N}=5$ cell culture preparations. D-F Rate of ATP consumption was indirectly calculated as the rate of increase in free magnesium that occurs upon ATP-hydrolysis, after blocking ATP production with oligomycin and iodoacetic acid. ATP depletion leads to cell collapse that is detected by a sudden increase in fluorescence. Representative traces of ATP consumption in cerebellum (D) and midbrain cells (E). (F) Time to cell collapse after blocking ATP production in cortical ( $N=5$ cell culture preparations) midbrain $(\mathrm{N}=4)$ and cerebellum ( $N=4$ cell preparations) neurons and astrocytes. ${ }^{*} p<0.05$; ${ }^{* *} p<0.01$; ${ }^{* * *} p<$ 0.001 . 
A

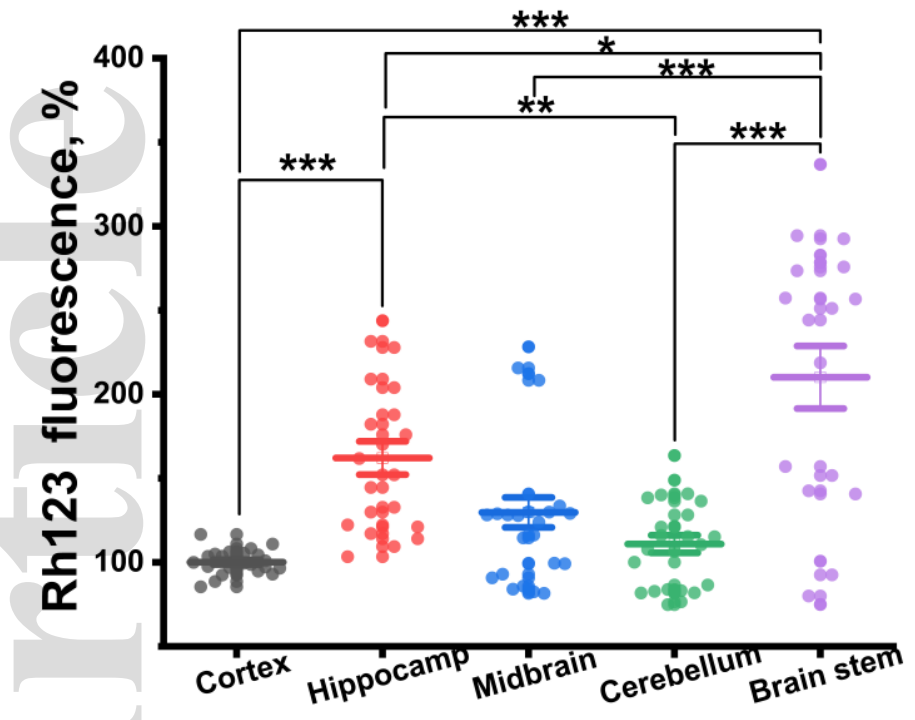

B

Hippocampus

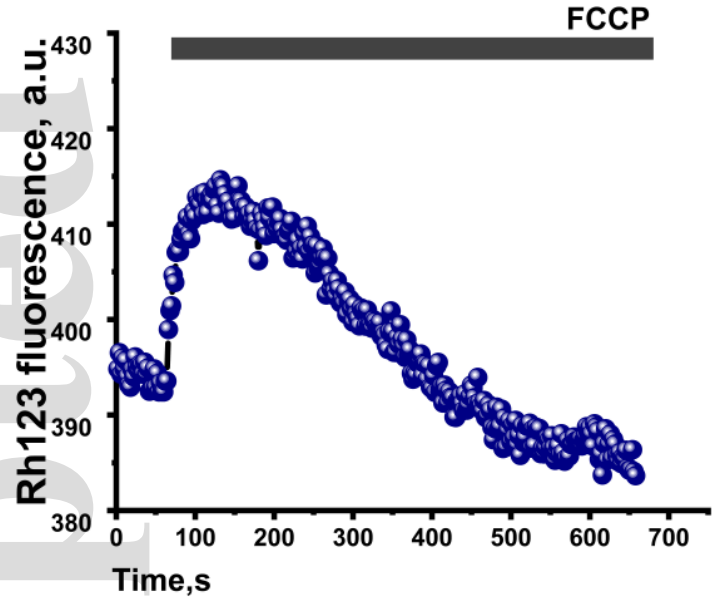

\section{Cerebellum}

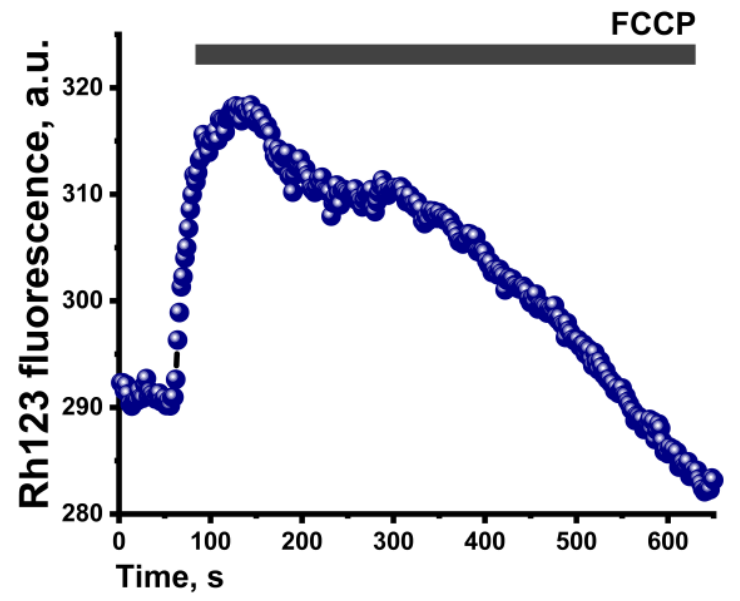

\section{Figure 1}



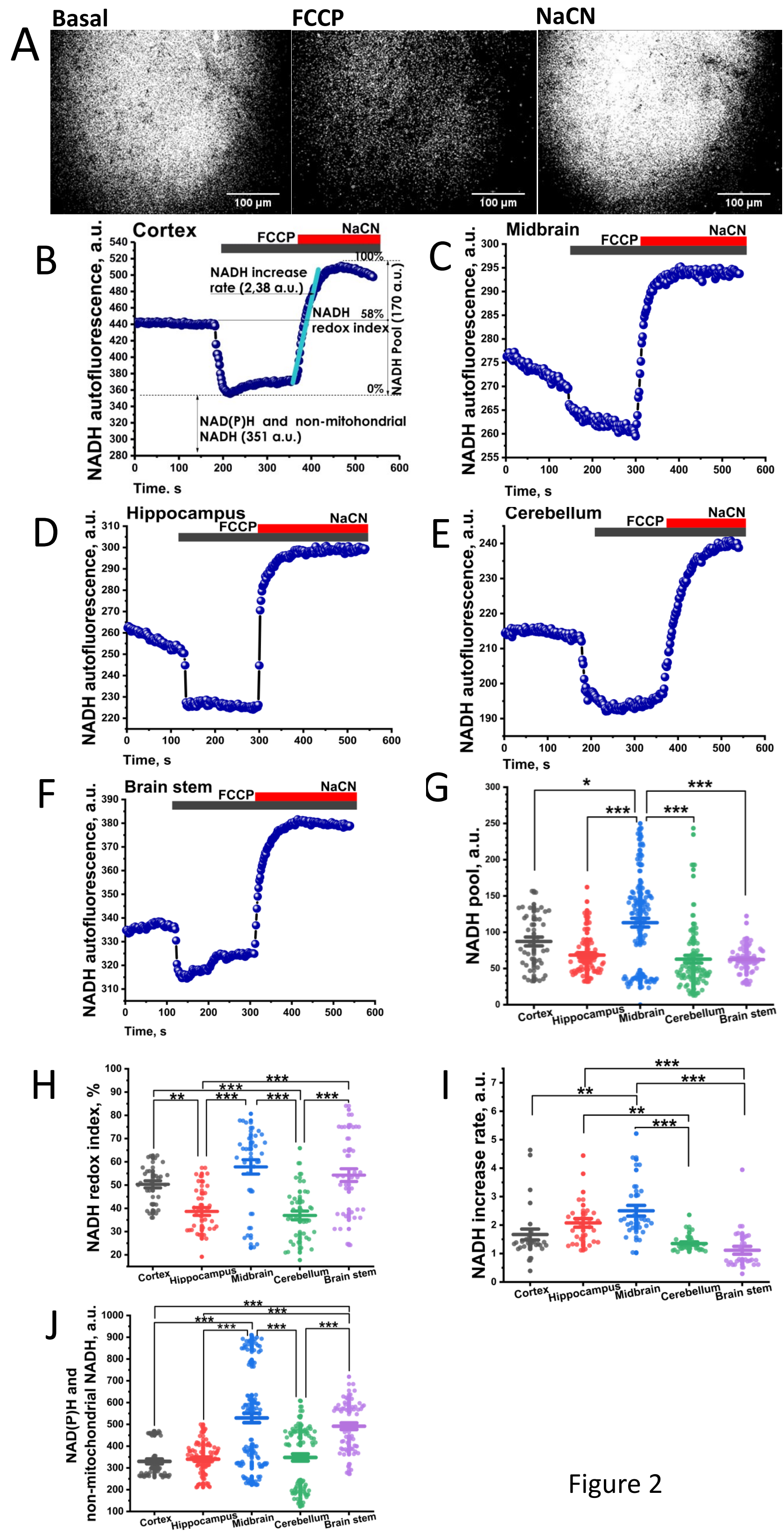

Figure 2 
A

Cerebellum

jnc_15239_f3.pdf

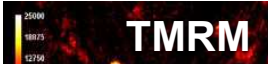

\section{Bright Field}

Z-projection (Stü m planes)

$20 \overline{\mathrm{m}}$

Focal Plane Neurons

$20 \overline{\mathrm{mm}}$

\section{wa.}

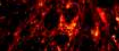

\section{Focal Plane Astrocytes}

${ }_{m=1}^{m s}$

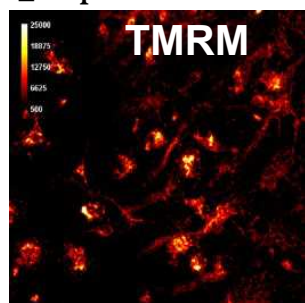

Midbrain

srightele

\section{Z-projection (sum planes)}

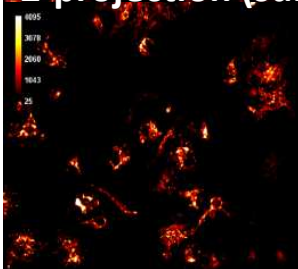

\section{Focal Plane Neurons}

$20 \mu \mathrm{m}$
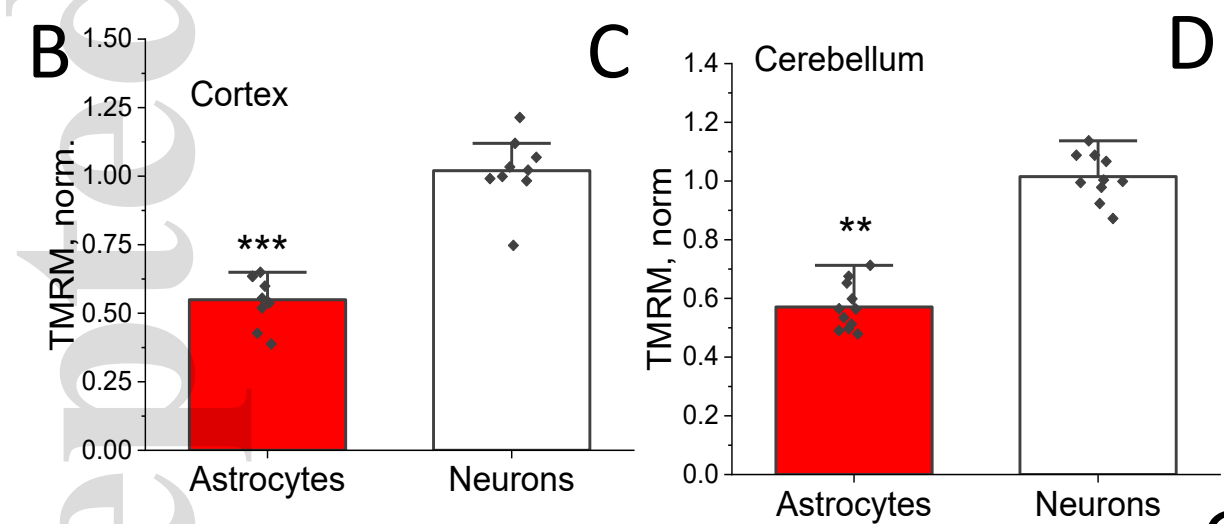

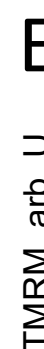

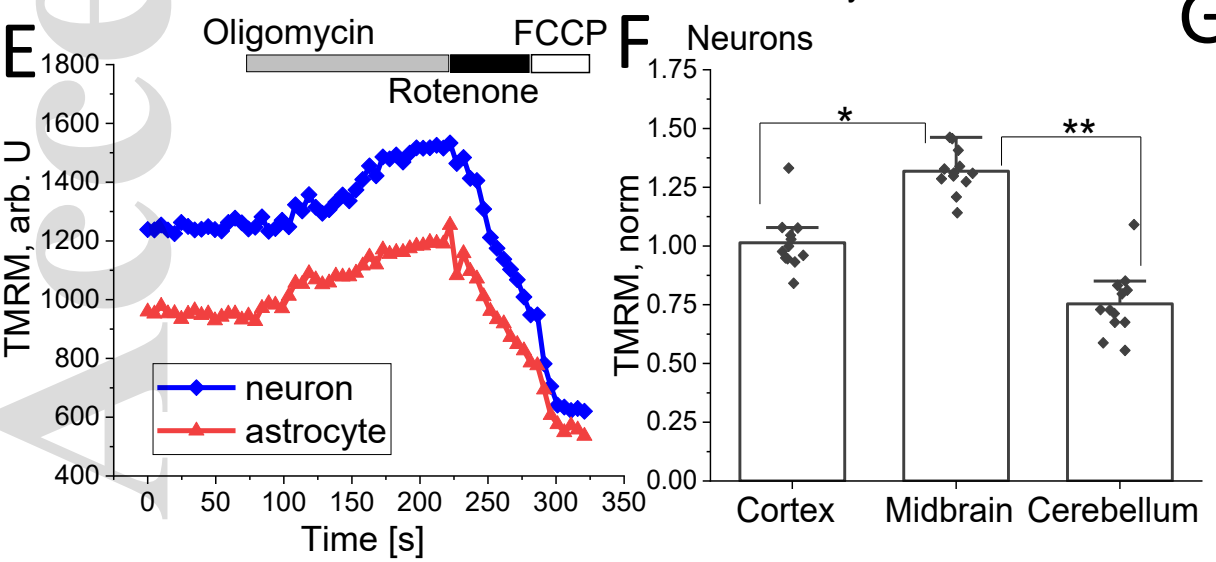

Focal Plane Astrocytes

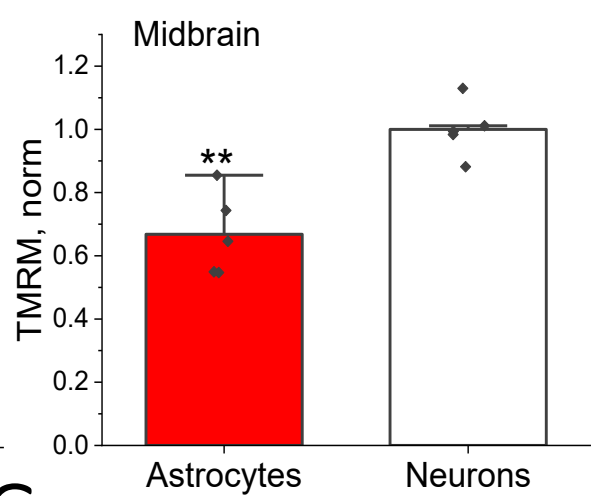

$\exists$

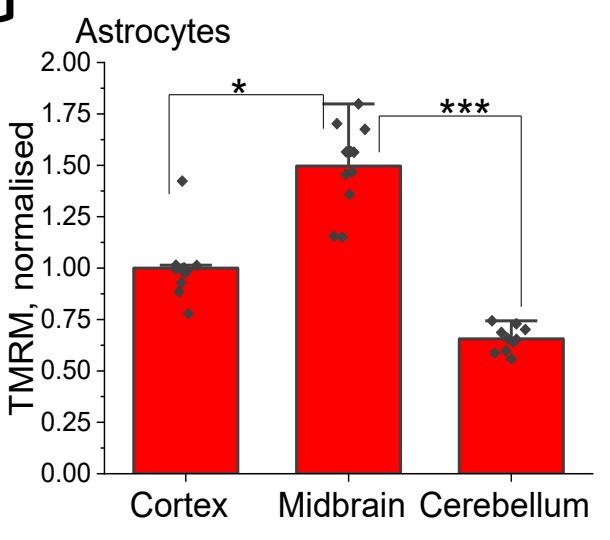

This article is protected by copyright. All rights reserved

\section{Figure 3}


A

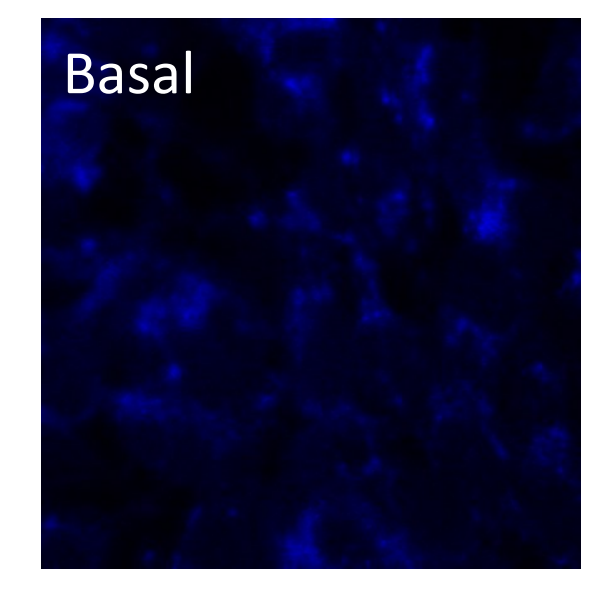

B

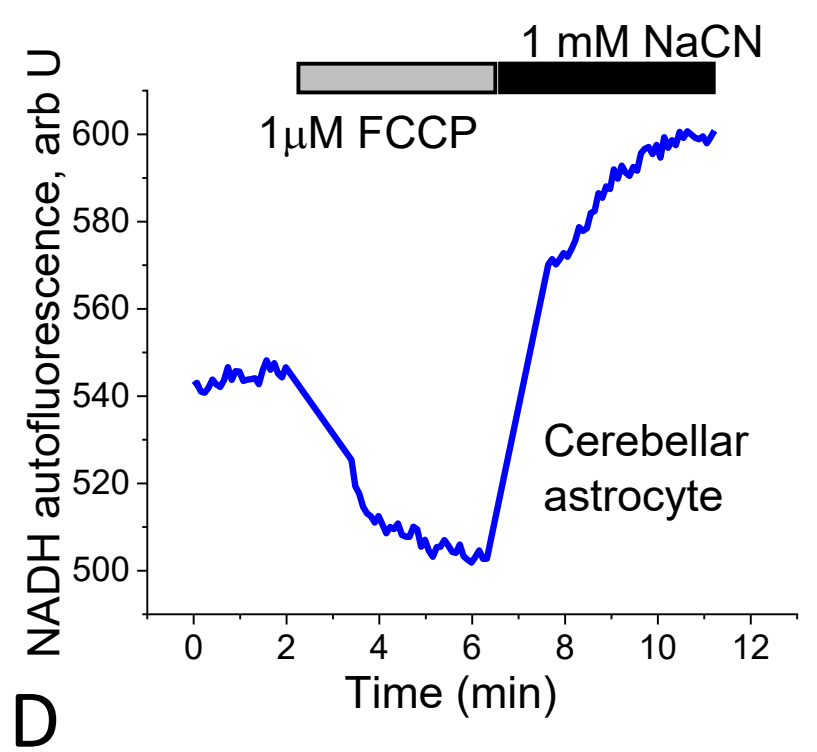

D

Astrosytes

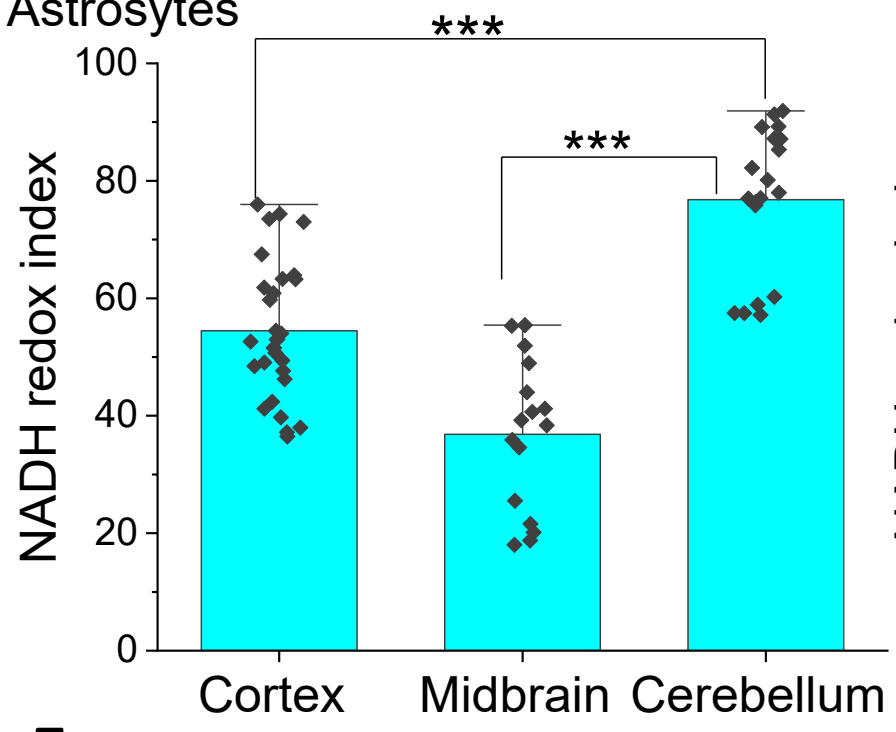

\section{C}
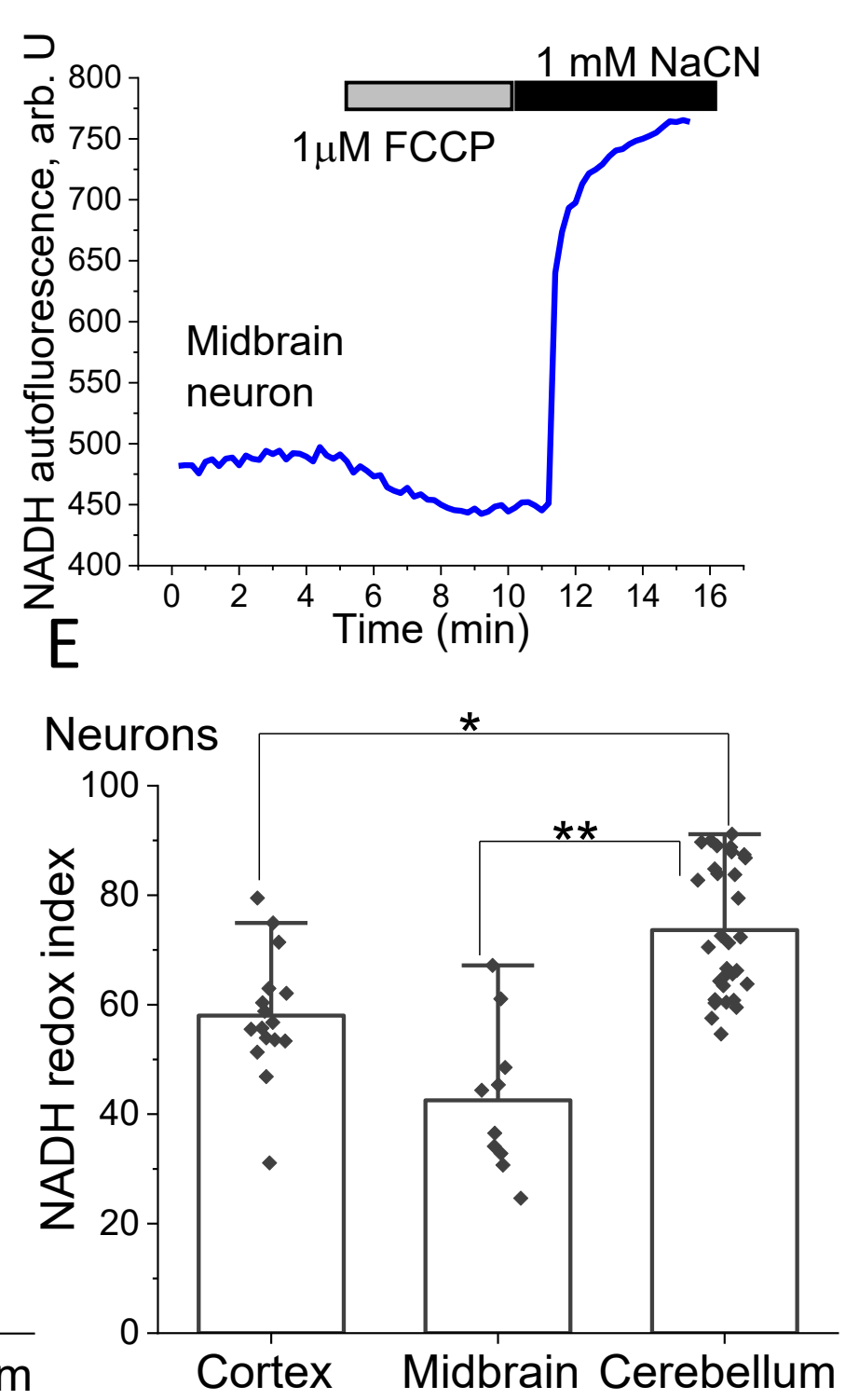

F

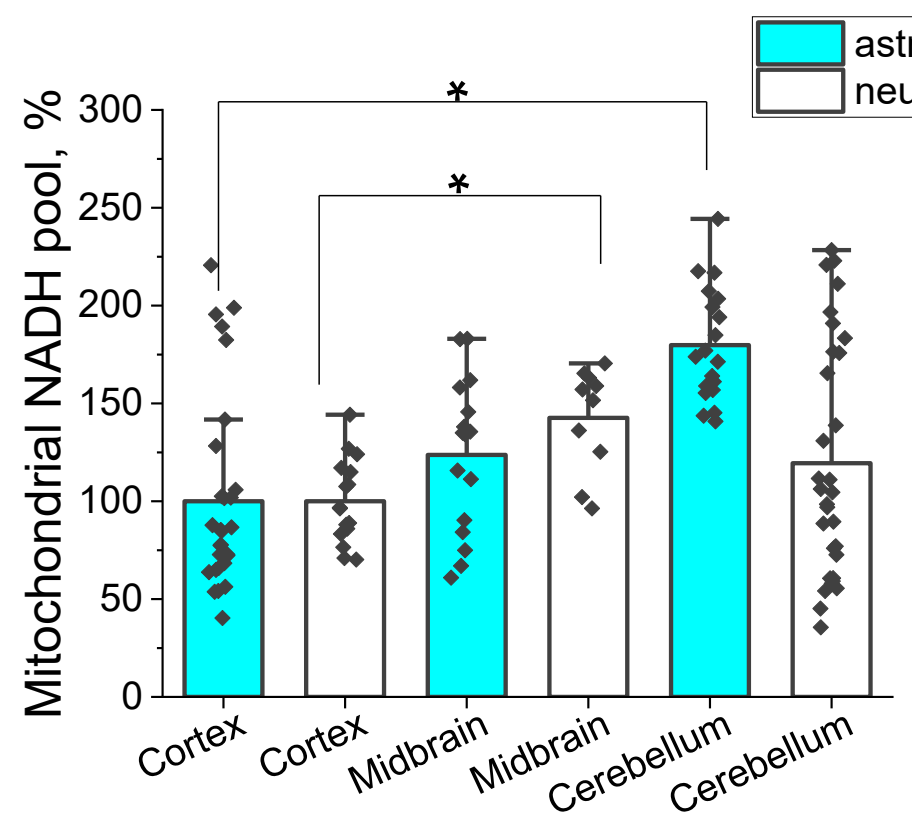

Figure 4 


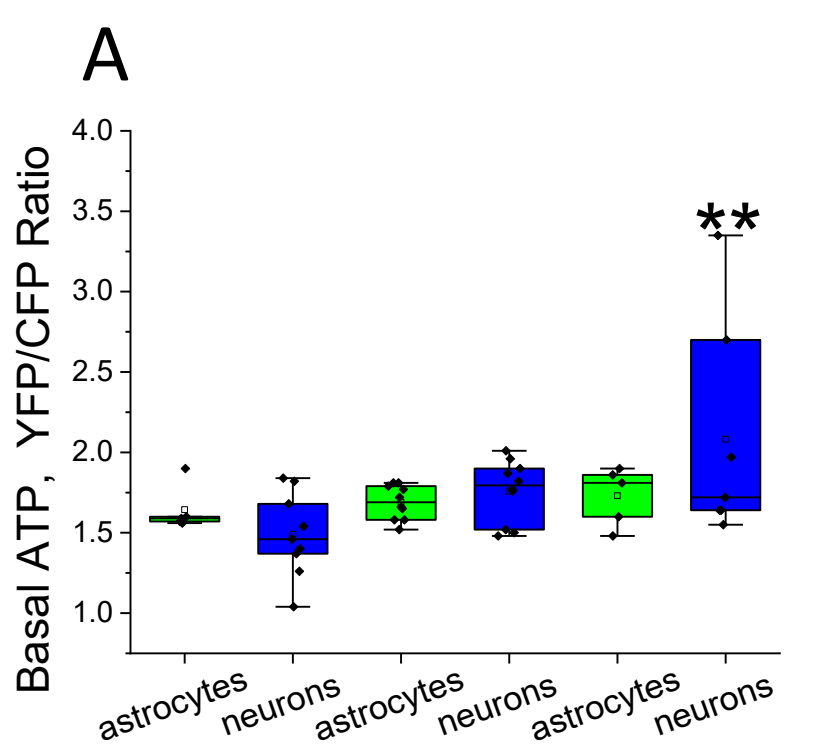

$\overline{\widetilde{\sigma}} B$

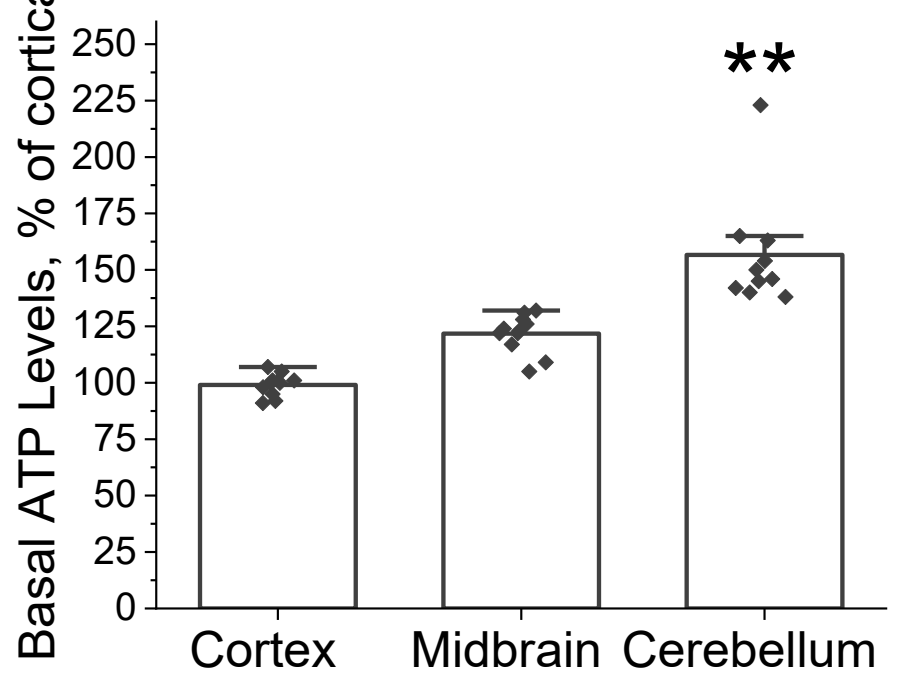

Cortex Midbrain Cerebellum
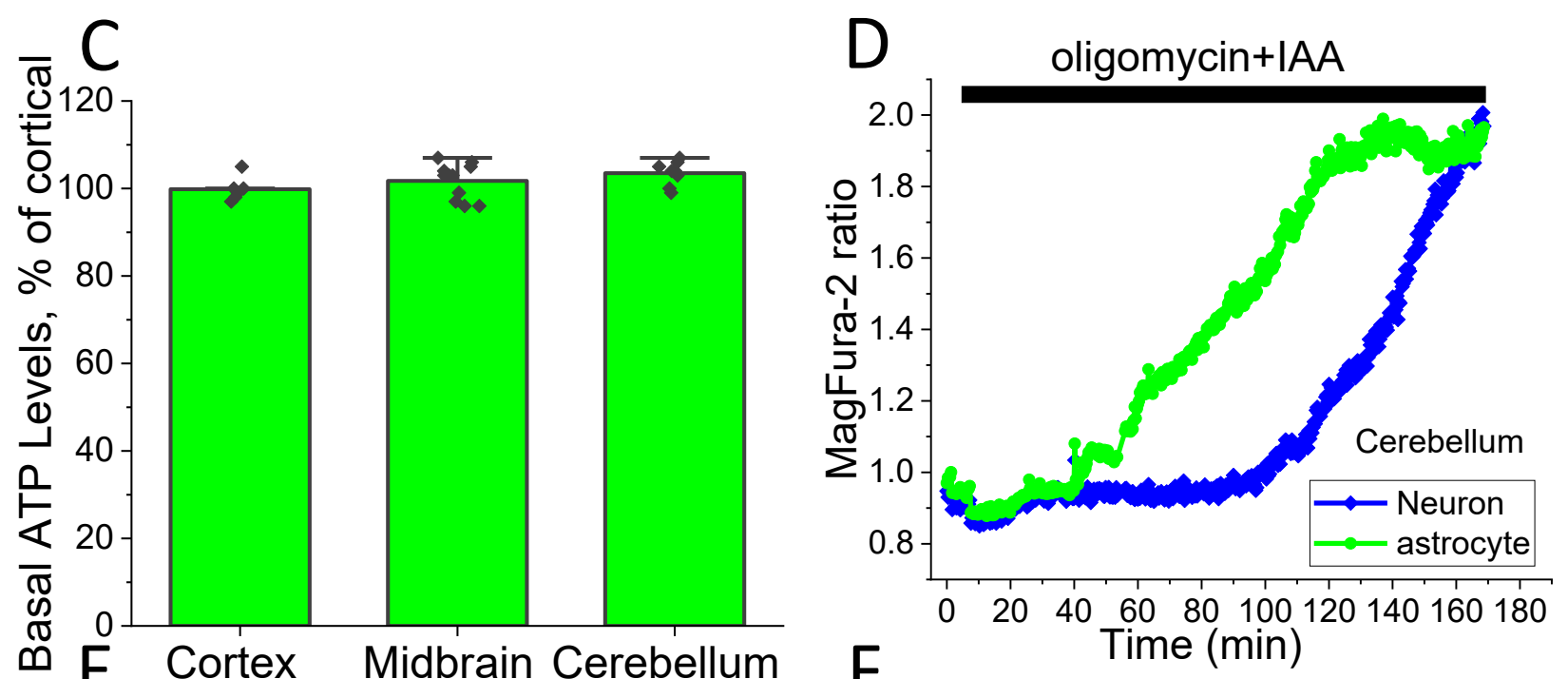

$\infty$ E Cortex Midbrain Cerebellum
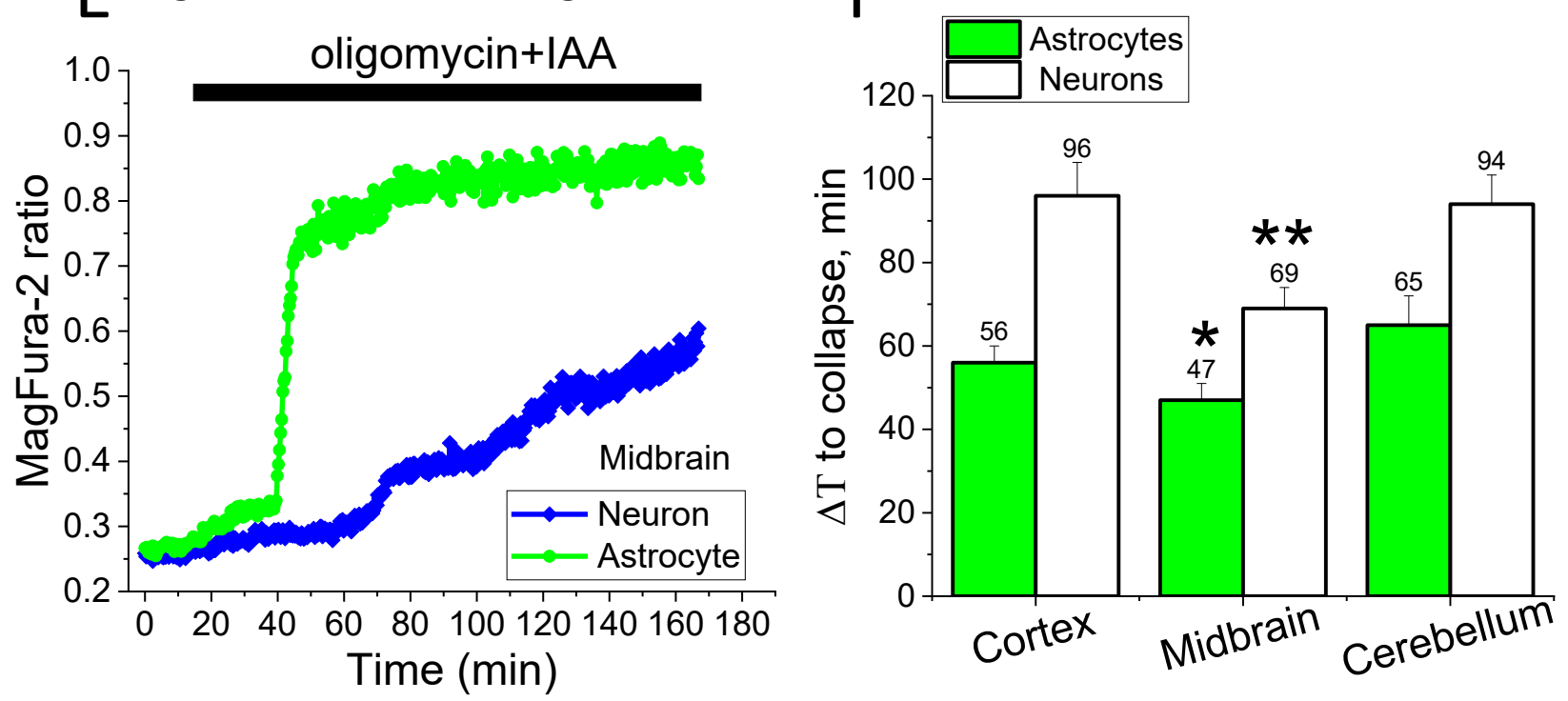

Figure 5 